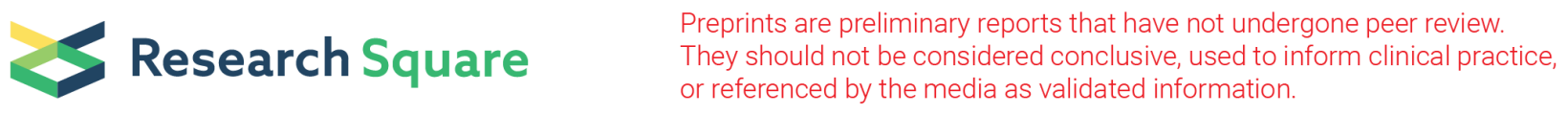

\title{
Eucalyptus Essential Oil Bioactive Molecules from against SARS-CoV-2 Spike Protein: Insights from Computational Studies
}

Arun Dev Sharma ( $\square$ arundevsharma47@gmail.com )

Lyalpur Khalsa College Jalandhar

Inderjeet Kaur

Lyalpur Khalsa College Jalandhar

\section{Research Article}

Keywords: COVID-19, Docking, Toruatone, Herbal Drug

Posted Date: January 4th, 2021

DOI: https://doi.org/10.21203/rs.3.rs-140069/v1

License: () (7) This work is licensed under a Creative Commons Attribution 4.0 International License. Read Full License 


\section{Abstract}

Background: SARS-CoV-2 (COVID-19), a positive single stranded RNA virus, member of corona virus family, is spreading its tentacles across the world due to lack of drugs at present. Being associated with cough, fever, and respiratory distress, this disease caused more than $15 \%$ mortality worldwide. Due to its vital role in virus replication, Spike (S) protein has recently been regarded as a suitable target for drug design. The present in silico docking study was designed to evaluate the effect of potent 12 bioactive molecules present in essential oils of eucalyptus plant leaves against Spike (S) protein from SARS-CoV-2. Till date there is no work is undertaken on in-silico analysis of these compounds against Spike (S) protein of SARS-CoV-2. In the present study, molecular docking studies were conducted by using Patchdock analysis.

Material and methods: Protein Interactions Calculator was used for protein interactions. In-silico absorption, distribution, metabolism, excretion and toxicity (ADMET) profile was also studied.

Results: The calculated parameters such as docking score indicated effective binding of all bioactive components especially Toruatone to COVID-19 S-protein. Interactions results indicated that, Spike (S) protein / Toruatone complexes forms hydrogen and hydrophobic interactions. Furthermore, our study also highlights the key finding that the S2 domain of COVID-19 spike glycoprotein potentially interacts with Toruatone. In-silico absorption, distribution, metabolism, excretion and toxicity (ADMET) studies provided guidelines and mechanistic scope for identification of potent anti-COVID 19 drug

Conclusions: Therefore, essential oil from eucalyptus may represent potential herbal treatment to act as COVID-19 Spike (S) protein inhibitor, a finding which must be validated in Vivo. However, further research is necessary to investigate their potential medicinal use.

\section{Introduction}

A new corona virus, 2019-n-CoV, caused a pandemic of respiratory disease (COVID-19) in Wuhan city, has since spread worldwide [1]. The virus has been called as SARS CoV2, as the RNA genome of virus is $82 \%$ similar to the SARS coronavirus. Presently, no particular treatment for COVID 19 is accessible and research concerning the therapy of COVID-19 is undergoing [2]. Nevertheless, the processes that have been applied remain restricted to supportive and defensive therapies, intended to avoid additional obstacles and damage to organs. Certain primary investigations have scrutinized possible blends that comprise anti-malarial drug hydroxychloroquine, and Anti Human Immuno deficieny virus (HIV) vaccines could be used for treating COVID19 infections. Besides using antiviral drugs, clinicians are utilizing MERS CoV and SARS CoV neutralizing antibodies with a specific target on the domain "S1" of spike protein (glycoprotein) of the COVID 19 [3].

Like other corona viruses, the outer membrane spike glycoprotein, is the major protein involved in interaction with specific targets of host cell (for instance adhesion factors, Ezrin, CD26, ACE2 and cyclophilins). All these targets are significant for adhesion and virulence. As far as entry of corona viruses is concerned, it is facilitated via transmembrane "S" glycoprotein that produces homo-trimer projecting from surface of virus [4]. The SARS-Cov "S" glycoprotein comprises a conserved Receptor Binding Domain (RBD) which identifies receptors of host cell such as Ezrin, CD26, ACE2 and cyclophilins. It is 1200 amino acids long protein belongs to class1viral fusion proteins and involves in binding with cell's receptor, pathogenesis and tissue tropism [5]. In the course of infection, the trimeric "S" protein is treated via proteases of host cell at the S1 or S2 cleavage site. Subsequent cleavage, also called it as priming, the protein is separated into two terminals: one is ' $\mathrm{S} 1$ ' ectodomain ( $\mathrm{N}$ terminal) that identifies similar surface receptor of cell and other is ' $\mathrm{S} 2$ ' membrane anchored protein ( $\mathrm{C}$ terminal) involved in entry of virus [6]. Therefore, by virtue of its key role, SARS-Cov 'S'protein is considered as an appropriate objective for developing viral inhibitor. Inhibition of SARS-Cov'S' protein activity would block replication of virus. Since in humans, not at all any proteases with comparable cleavage specific are recognized, so inhibitors are improbable to be considered as toxic.

The invention of new drugs as therapeutic agents is a tedious and expensive process. Traditional methods can take about 12-14 years with lot of expenditure to bring a drug into market [7]. So as too overcome these challenges, multi disciplinary approaches are required in the process of drug discovery. In drug discovery processes, in-silico designing of drug is a form of computer based modeling which is very useful. This method offers advantage to deliver a new drug in fast and cost-effective manner. As of this date, the process of drug designing has been advanced with various bioinformatics tools which help in molecular docking analysis, protein- protein/ligand interaction, virtual screening and de-novo synthesis and in silico ADMET prediction.

From ancient time, medicinal plants are beneficial in the field of drug therapeutics as they are safer alternatives being utilized by humans for centuries [8]. Previously, many of the new drug formulations are derived from natural products. Our present study focuses on the in silico analysis of essential oil present in Eucalyptus plants. Various plant parts like flowers, leaves, roots and fruits have been used in various system of medicine for the past 2000 years to treat numerous diseases such as fever, skin infection, bleeding piles, asthma, cold, cough and inflammation. Hence, great attention has been paid to plant secondary metabolites as therapeutic medicines. Essential oils due to having antioxidant, antimicrobial and anti-viral bioactivities, immensely used in cosmetic, pharmaceutical industries. Essential oils are complex mixture of volatile elements such as flavonoids, alkaloids, aromatic compounds and terpenes. Eucalyptus essential oil has been empirically used as antimicrobial agents, but little is known about its antiviral potential [9]. We hypothesize that bioactives from essential oil have the capability to prevent infection of COVID-19. Therefore the research objective of the current study was in-silico analysis and comparative molecular docking studies pertain to bioactive molecules in relation with S-protein protein. The outcomes of the current study would provide researchers with prospects to recognize the accurate drug to combat COVID19. To further estimate the drug ability of this phytochemical, the present study report their ADMET profiles and potential biological activities using various in silico bioinformatics tools.

\section{Materials And Methods}

Proteins/Macromolecules 
SARS-CoV-2 S-protein structures were obtained from PDB (https://www.rcsb.org/). The 3-dimensional (3D) structure of Eucalyptus essential oil bioactive molecules (Figure 1) were obtained from PubChem (https://pubchem.ncbi.nlm.nih.gov/).

\section{Ligand and Drug Scan}

Eucalyptol was retrieved from PubChem (https://pubchem.ncbi.nlm.nih.gov/). Lipinski's rule of five was used to calculate drug like properties. SWISSADME prediction tool was used to find Lipinski's rule of five (http://www.swissadme.ch/) and to find out ADMET (Absorption, Metabolism, Toxicity and Excretion) properties. Bioactivity analysis was carried out using molinspiration tool (https://www.molinspiration.com/cgi-bin/properties). Bioactivity was based on following score: if the bioactivity score is $(>0)$, then it is active, if $(-5.0-0.0)$ then moderately active, if $(<-5.0)$ then inactive.

\section{Binding Mode of Docked Complexes}

Plip tool was use to find out residues involved in 2-D interactions (https://projects.biotec.tu-dresden.de/plip-web/plip/index/).

\section{Active Sites Determination}

CASTp too, was used to find out amino acid residues present in the active site of a protein (http://sts.bioe.uic.edu/castp/index.html?201l)

\section{Result And Discussion}

Traditionally, the discovery of new therapeutic drugs is a tedious and expensive process which generally takes 12-14 years with a lot of money to bring drug into market. With the purpose of overcoming these problems a lot many multidisciplinary approaches are used to discover new drug. In drug discovery processes, in silico designing of drug is a practice of computer based modeling which is very useful. In the field of drug discovery, medicinal plants are advantageous as they are utilized as a safe herbal alternative by humans for centuries. The sources of several of the active constituents of medicines and novel drugs are obtained from natural products.

Coronaviruses (CoVs) are a group of viruses that infects humans and animals. CoVs infections affect animals by several means such as: cold, fever, digestive, respiratory and liver systems of animals and humans. Enveloped viruses get enterinto their respective host cells through membrane fusion process that is facilitatedvia a precise fusion, or"S" protein (virus encoded), and embedded in the envelope of the virus [4]. Such proteins are presently clustered into three diverse structural classes, with the so called class I fusion proteins usually primed for fusion activation through proteolytic cleavage. It is the S-protein present in the CoV related with the Severe Acute RespiratorySyndrome (SARS), which could be gain access to in PDB and was proposed to be a possible drug target for 2019-nCov [5]. In several viruses, S-protein play crucial roles in entry of virus into host cells; therefore, S-proteins are frequently used as protein targetsthroughout the development of antiviral therapeutics. The coronavirus spike proteins (S) play animportant role in the initial stepsof viral infection, with the S1 domain responsible for receptors binding and the S2 domain region (aa570-aa1278),facilitating membrane fusion. As cited by earlier,S-proteins signify key targets for the inhibition of viral replication, and the protein sequences of the SARS-CoV S-protein and the 2019-nCoV S-protein are $91 \%$ identical, hence host proteases could be exploited as probable therapeutic targets [4].

In the present study, we performed in silico analysis of eucalyptus essential oil component eucalyptol (1-8 cineole) against S-protein protein of CoVID-19. In modern drug discovery process, molecular docking is a widely used computational method to predict the binding mode and binding affinity of ligands with the target receptor protein. The efficacy of the docked complex was estimated on the basis of two important criteria's: The lowest binding energy and the interaction of the ligand with the active site amino acids residues. A ligand experiences either hydrophobic interactions or $\mathrm{H}$-bonding or both while docking in the active site. The results of docking can be used to find the best inhibitors for specific target proteins and thus to design new drugs. We followed the structural biology aspects which focus on the availability and retrieval of a S-protein receptor structure from PDB database.

Molecular docking using patchDock docking tool that was used to find out interaction of inhibitor bioactive compounds of essential oil with S- protein revealed 20 different poses based on the dock score and area with highest score values indicated maximum binding affinity (data not shown). Among all molecules, Toruatone exhibited highest affinity with S-protein with maximum docking score followed by Phenethyl phenylacetate (Table 1). The high affinity of drug compounds depend on amount and the type of bonding that occur with the active site of the protein. As indicated in Figure 2, Toruatone forms many chemical bonds with S2 domain of S protein, including hydrogen bonds and hydrophobic bonds. S-protein comprises two functional subunits responsible for binding to the host cell receptor (S1 unit) and fusion of the viral and cellular membrane (S2) subunit [5]. The interaction of Toruatone in the binding pocket of S2-protein domain was mediated by hydrophobic interactions via PRO589 and LEU577 with S2-protein domain at atomic distances of 3.68 and 3.11 (Figure 2). The Toruatone showed full fitness within active site amino acids of S2 domain of S-protein of SARS-CoV-2. According to the Plip server and Biovia studio results as shown in Table 1, S-protein protein formed complexes with bioactive ligands molecules from eucalyptus essential oil by forming various interactions as hydrophobic residues interactions, and hydrogen bond. For example, Eucalyptol formed hydrophobic interactions with ALA1016. Result of the present study shown that LEU761/864 and GLY613/667 were critical residues for binding of Jensenone to S-protein via hydrophobic and hydrogen bond interactions, respectively. GLN1002 and THR1009 participated in hydrophobic interaction with Citronellal and Geranyl acetate. It was postulated that these ligand-protein interactions might inhibit the formation of the S2 motif of S- protein. Coronavirus trafficking into and hijacking the host cell is primarily driven by the C-terminal S2 domain of spike glycoprotein that interacts with several host cell proteins [3]. It was postulated that COVID-19 S-protein becomes closed upon binding with eucalyptol which in turn brings conformational changes of the S-protein and stop initiation of the fusion reaction responsible for its insertion into the host cell membrane [Song et al., 2018]. Antiviral bioactivities of many compounds like flavonoids, terpenoids and phenolics from essential oils, have been reported. Further as indicated in Table 1, almost all the essential oil molecules formed hydrogen bonds and hydrophobic interactions with active site amino acids of S-protein of COVID-19. Hydroxy groups $(-\mathrm{OH})$, ketone groups $(=0)$ and ether groups $(-0-)$ in eucalyptol compounds are predicted to play roles amino acid residue interactions at the active site of COVID-19 S-protein. Further studies may help to understand the role of these residues in drug 
binding mechanism. Structural flexibility is one of the important physical properties that affect protein conformation and function. In this context, an inhibitor by binding to a protein can alter its flexibility and decrease its enzymatic activity. Further studies may help to understand the role of these residues in drug binding mechanism.

Pharmacokinetic analysis using ADMET properties was studied. Eucalyptol ligand used in this study did not violate Lipinski's rule of five [2]. Eucalyptol scanning results are illustrated in Table 2. Topological polar surface area (TPSA) value was considerably less than $90^{\circ} \mathrm{A}$ squared. It indicated good permeability of eucalyptol cell membranes to enter Blood Brain Barrier (BBB). Pharmacokinetics parameters and ADMET factors are key parameters for success of mostly drugs during clinical trials [9]. Blood Brain Barrier (Log BB) permeability which was good with eucalyptol. The eucalyptol showed positive results for BBB profile. Log $\mathrm{P}_{\mathrm{o} / \mathrm{w}}$ (lipophilicity indicator, octanol-water partition coefficient) was 2.67, indicating eucalyptol was optimal BBB penetration. Eucalyptol did not shown any affinity with P-glycoprotein (p-gp), a efflux transporter located at BBB. GI (Gastrointestinal tract absorption) of eucalyptol was high (Table 2). In order to exert a toxic effect, drug molecules have to be absorbed from intestinal tract in the body. Further, eucalyptol shown non inhibitory activity against cytochrome P series (CYP1-3) of enzymes, involved in liver detoxificatyion of toxins from body. 3D molecular structures showing Molecular Lipophilicity Potential (MLP) and Polar Surface Area (PSA) are also shown in Fig. 4. MLP is convenient property to rationalize numerous molecular ADME characteristics (for example: plasma-protein binding or membrane penetration). Analysis of 3D distribution of hydrophobicity on molecular surface is predominantly helpful when explaining differences is observed in ADME properties of molecules with the same logP.

\section{Conclusion}

Due to non approved drugs at present Currently, SARS-CoV-2 has emerged in the human population, in China, and is a potential threat to global health, worldwide. Currently, the main target for COVID-19 treatment primarily act on the S-protein. The aim of this study was to examine Bioactive molecules from eucalyptus essential oil that may be used to inhibit the SARS-CoV-2 infection pathway. Therefore, we suggested that Eucalyptol may represent potential treatment options, and found in medicinal plants that may act as potential inhibitors of COVID-19 S-protein. However, further studies should be conducted for the validation of these compounds using in vitro and in vivo models to pave a way for these compounds in drug discovery.

\section{Declarations}

\section{Conflict of interest}

Authors declares no conflict of interest

\section{References}

1. Huang, C., Wang Y. and Li, X., et al., 2020. Clinical features of patients infected with 2019 novel coronavirus in Wuhan, China, Lancet. 395 , 497-506 .

2. Lipinski, C.A. 2004. Lead- and drug-like compounds: the rule-of-five revolution. Drug Discovery Today, Technologies 1, $337-341$.

3. Liu, X. and Wang X.J. 2020. Potential inhibitors against 2019-nCoV coronavirus M protease from clinically 13 of 14 approved medicine, Genet. Genomics, doi: 10.1016/j.jgg.2020.02.001.

4. Lu, H. 2020. Drug treatment options for the 2019-new coronavirus (2019-nCoV), Trends, doi:10.5582/bst.01020.

5. Millet, J.K., Kien, F. and Cheung, CY., et al. 2012. Ezrin interacts with the SARS coronavirus spike protein and restrains infection at the entry stage, Plos One:; 7

6. Sharma, A.D., and Kaur, I. 2020. Molecular docking studies on Jensenone from eucalyptus essential oil as a potential inhibitor of COVID 19 corona virus infection, Research and Reviews in Biotechnology and Biosciences, 1, 59-66 (2020).

7. Shukla, A., Sharma, P., Prakash, O., Singh, M. and Kalani, K., et al. 2014. QSAR and Docking Studies on Capsazepine Derivatives for Immunomodulatory and Anti- Inflammatory Activity, PLoS ONE 9, e100797.

8. Song, W.F., Gui, M. and Wang, X., et al. 2018. Cryo-EM structure of the SARS coronavirus spike glycoprotein in complex with its host cell receptor ACE2, PLoS Pathog.14, e1007236.

9. Yang, L., Wen, K.S., Ruan, X., Zhao, Y.X., Wei, F. and Wang, Q. 2018. Response of plant secondary metabolites to environmental factors, Molecules, 23, 126, doi: 10.3390/molecules23040762.

10. Zakaryan, H., Arabyan, E., Oo, A. and Zandi, K. 2017. Flavonoids: promising natural compounds against viral infections. Virol., 162: 2539-2551, , doi: 10.1007/s00705-017-3417-y.

\section{Tables}

Table 1: Docking analysis of essential oils molecules with spike protein 


\begin{tabular}{|llllll|}
\hline Model name & & & \multicolumn{3}{l|}{ Interacting residues and length (4 A $)$} \\
\hline Eucalyptol & Score & Area & ACE & & ALA11016,1020 \\
\hline Citronellol & 3122 & 376.90 & -60.19 & & PRO655, VAL772, LEU861 \\
\hline Citronellal & 3792 & 423.20 & -85.35 & GLN314, 613 & GLY1002, THR1009 \\
\hline Phenethyl phenylacetate & 4846 & 536.40 & -162.45 & SER 513 & ILE312, THR768, VAL772 \\
\hline Geranyl acetate & & & & GLN613 & \\
\hline Limonene & 4396 & 485.00 & -86.73 & GLN1002 & GLN1002, THR1009 \\
\hline 4-terpineol & 3340 & 385.40 & -104.30 & - & ALA1016,1020 \\
\hline P-cymene & 3476 & 386.30 & -73.80 & THR1009 & LEU390, 517,546, ALA522, PHE565 \\
\hline Alpha-pinene & 3440 & 376.80 & -187.94 & - & ALA1016, 1020 \\
\hline Toruatone & 3476 & 381.10 & -94.02 & - & PR0989, LEU977 \\
\hline Jensenone & 5324 & 570.20 & -134.81 & ASP745, ARG1000 & PR0655, VAL772, ASP775, LEU 761,864 \\
\hline Cis-p-2-Menthen-1-ol & 3162 & 473.70 & -114.97 & SER596, GLY613, 667, LYS733 & LEU390,517, ASN544 \\
\hline
\end{tabular}

Table 2: Pharmacokinetics properties of Bioactive molecules from Eucalyptus essential oil

\begin{tabular}{|c|c|c|c|c|c|c|c|c|c|c|c|c|}
\hline & \multirow[t]{2}{*}{ Molecule } & \multicolumn{5}{|c|}{ Lipinski's rule of five } & \multicolumn{6}{|c|}{ ADMET properties } \\
\hline & & $\begin{array}{l}\text { Molecular } \\
\text { weight }\end{array}$ & $\begin{array}{l}\text { Num. H- } \\
\text { bond } \\
\text { acceptors }\end{array}$ & $\begin{array}{l}\text { Num. } \\
\mathrm{H}- \\
\text { bond } \\
\text { donors }\end{array}$ & $\begin{array}{l}\text { Molar } \\
\text { refractivity }\end{array}$ & $\begin{array}{l}\text { lipophilicity } \\
\text { logP }\end{array}$ & $\begin{array}{l}\quad \text { GI } \\
\text { absorpti } \\
\text { on }\end{array}$ & $\begin{array}{l}\text { Consensus } \\
\text { Log Po/w }\end{array}$ & $\begin{array}{l}\text { TPSA } \\
\AA^{2}\end{array}$ & $\begin{array}{l}\text { BBB } \\
\text { permeant }\end{array}$ & $\begin{array}{l}\text { P-gp } \\
\text { substrate }\end{array}$ & C) \\
\hline \multirow{3}{*}{1} & & & 1 & & & & & & 9.23 & Yes & No & $\mathrm{Nc}$ \\
\hline & EUCALYPTOL & $\square$ & & 0 & 47.12 & 2.58 & High & 2.67 & & & & \\
\hline & & $\begin{array}{l}154.25 \\
\mathrm{~g} / \mathrm{mol}\end{array}$ & & & & & & & & & & \\
\hline 2 & CITRONELLOL & $\begin{array}{l}156.27 \\
\mathrm{~g} / \mathrm{mol}\end{array}$ & 1 & 1 & 50.87 & 2.72 & High & 2.92 & 20.23 & Yes & No & $\mathrm{Nc}$ \\
\hline 3 & CITRONELLAL & $\begin{array}{l}154.25 \\
\mathrm{~g} / \mathrm{mol}\end{array}$ & 1 & 0 & 49.91 & 2.49 & High & 2.94 & 17.07 & Yes & No & $\mathrm{Nc}$ \\
\hline 4 & $\begin{array}{l}\text { PHENETHYL } \\
\text { PHENYLACETATE }\end{array}$ & $\begin{array}{l}240.30 \\
\mathrm{~g} / \mathrm{mol}\end{array}$ & 2 & 0 & 71.60 & 2.95 & High & 3.50 & 26.30 & Yes & No & $\mathrm{Nc}$ \\
\hline 5 & $\begin{array}{l}\text { GERANYL } \\
\text { ACETATE }\end{array}$ & $\begin{array}{l}196.29 \\
\mathrm{~g} / \mathrm{mol}\end{array}$ & 2 & 0 & 60.13 & 2.83 & High & 3.21 & 26.30 & Yes & No & $\mathrm{Nc}$ \\
\hline 6 & LIMONENE & $\begin{array}{l}136.23 \\
\mathrm{~g} / \mathrm{mol}\end{array}$ & 0 & 0 & 47.12 & 2.72 & Low & 3.37 & 0.00 & Yes & No & $\mathrm{Nc}$ \\
\hline 7 & 4-TERPINEOL & $\begin{array}{l}154.25 \\
\mathrm{~g} / \mathrm{mol}\end{array}$ & 1 & 1 & 48.80 & 2.51 & High & 2.60 & 20.23 & Yes & No & $\mathrm{Nc}$ \\
\hline 8 & P-CYMENE & $\begin{array}{l}134.22 \\
\mathrm{~g} / \mathrm{mol}\end{array}$ & 0 & 0 & 45.99 & 2.51 & Low & 3.50 & 0.00 & Yes & No & $\mathrm{Nc}$ \\
\hline 9 & ALPHA-PINENE & $\begin{array}{l}136.23 \\
\mathrm{~g} / \mathrm{mol}\end{array}$ & 0 & 0 & 45.22 & 2.63 & Low & 3.44 & 0.00 & Yes & No & $\mathrm{Nc}$ \\
\hline 10 & TORUATONE & $\begin{array}{l}280.36 \\
\mathrm{~g} / \mathrm{mol}\end{array}$ & 4 & 0 & 80.47 & 3.22 & High & 3.35 & 44.76 & Yes & No & $\mathrm{Nc}$ \\
\hline 11 & JENSENONE & $\begin{array}{l}266.25 \\
\mathrm{~g} / \mathrm{mol}\end{array}$ & 6 & 3 & 67.90 & 0.76 & High & 1.30 & 111.9 & No & No & $\mathrm{Nc}$ \\
\hline 12 & $\begin{array}{l}\text { CIS-P-2- } \\
\text { MENTHEN-1-OL }\end{array}$ & $\begin{array}{l}154.25 \\
\mathrm{~g} / \mathrm{mol}\end{array}$ & 1 & 1 & 48.50 & 2.46 & High & 2.46 & 20.23 & Yes & No & $\mathrm{Nc}$ \\
\hline
\end{tabular}




\section{Figures}

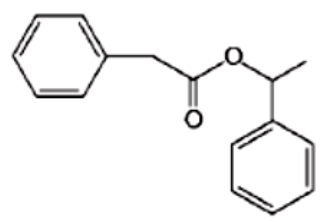

phenylethyl phenylacetate

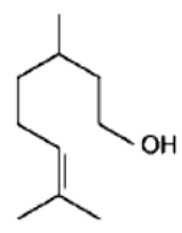

citronellol

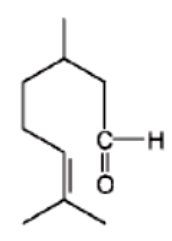

citronellal

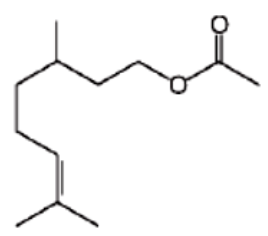

geranyl acetate<smiles>CC(C)C1C=CC(C)(O)CC1</smiles>

$\delta$-2-p-menthen-ol-(1)

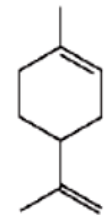

limonene

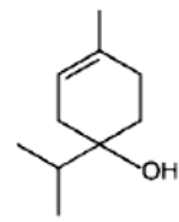

terpinen-4-ol<smiles>CC1=CCC(C(C)C)=CC1</smiles>

$\gamma$-terpinene

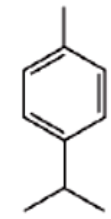

p-cymene

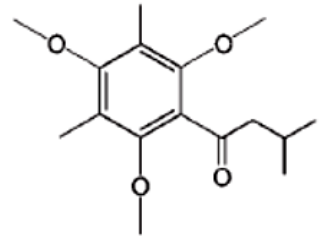

torquatone

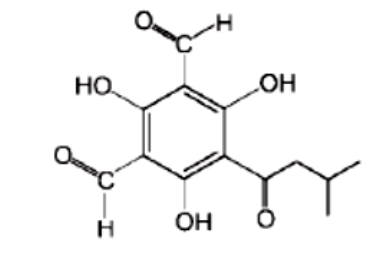

jensenone

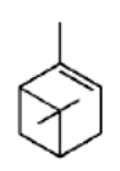

$\alpha$-pinene

Figure 1

Molecular structures of main substances in essential oil 

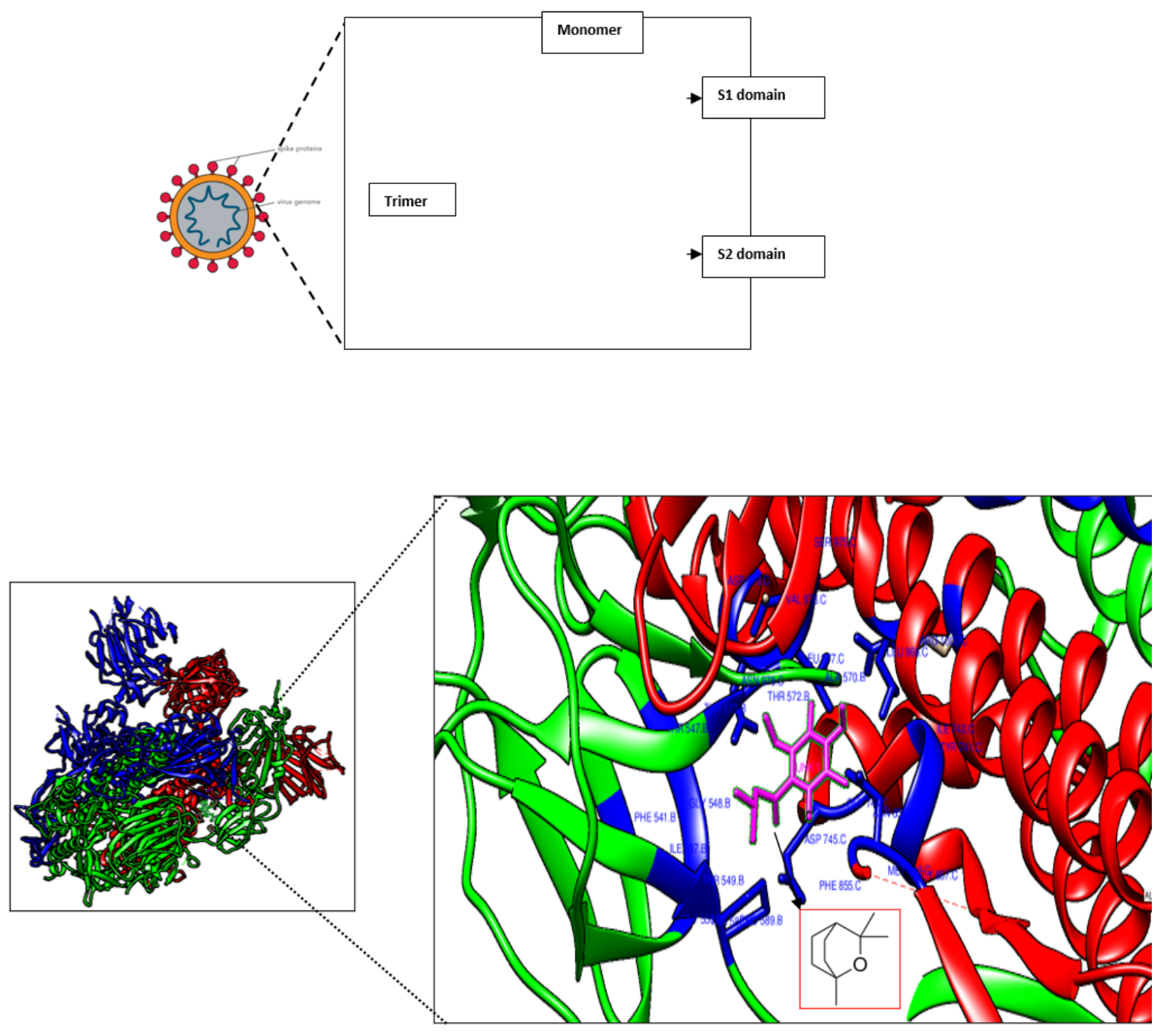

Figure 2

Three-dimensional docked structure of SARS-CoV-2 S-protein with Toruatone. Protomers of the trimer is shown in light blue, green and the other one in red. Domains are labeled by S1and S2 Roman numbers. 

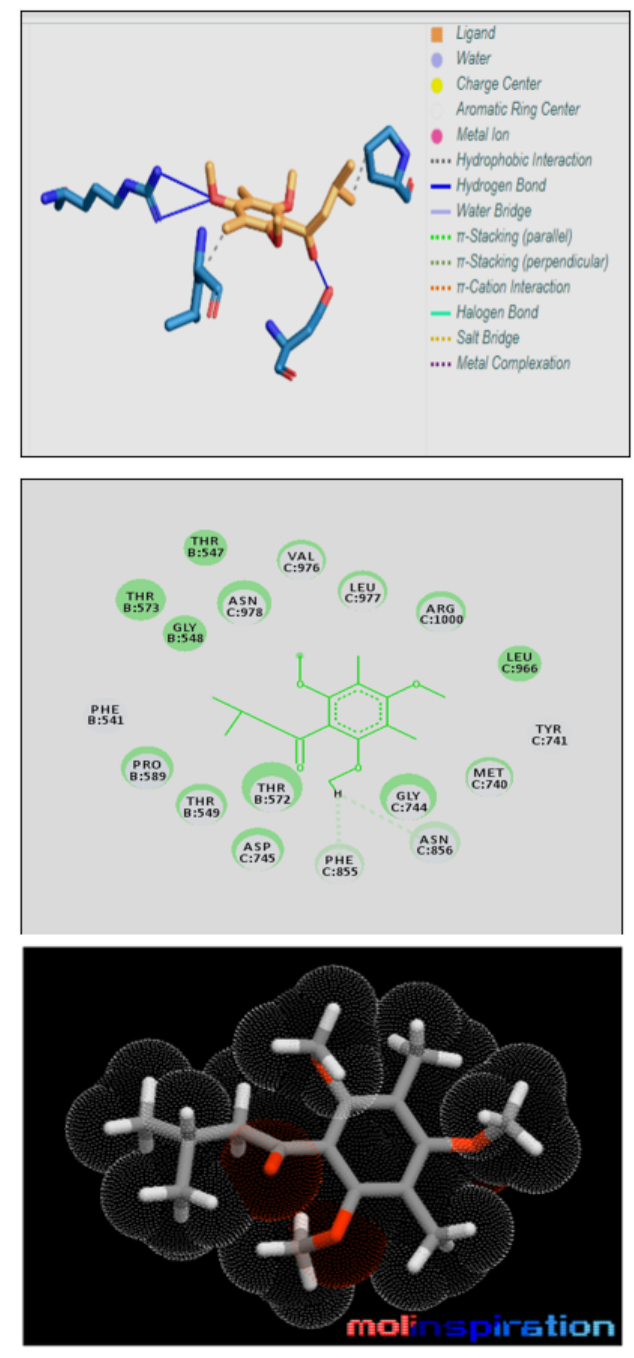

Figure 3

Molecular interactions and molecular lipophilicity potential of Toruatone with SARS-CoV 2 protein 